\title{
Freunde führen einander - Der kommunalpolitische Dialog mit dem „Islam“ im Modus einer Gouvernementalität der Freundschaft
}

\author{
Jan Winkler \\ Institut für Geographie, Friedrich-Alexander-Universität Erlangen-Nürnberg, Nuremberg, Germany
}

Correspondence to: Jan Winkler (jan.winkler@fau.de)

Received: 9 September 2016 - Revised: 3 May 2017 - Accepted: 29 May 2017 - Published: 14 July 2017

\begin{abstract}
Kurzfassung. Drawing on Foucault's notion of governmentality and on ethnographic fieldwork in a German city, this paper addresses practices of governing "Muslims" and "Islam" at the level of local politics. It conceptualises these practices as a governing through friendship, that is, a type of "liberal" conduct-of-conduct that focuses on trust-building and practised "togetherness". The paper analyses techniques of governance and processes of subjectivation, putting an emphasis on how emotionality becomes integrated into governmental practices. I show how governing through friendship unfolds in the spatio-political context of a local dialogue with "Islam", how the latter is linked to the interfaith paradigm and thus becomes a religiously-sensitive, post-secular political technology in which religious sensibilities are becoming elements of governmentality.
\end{abstract}

\section{Einleitung}

Die vorliegende Studie analysiert, wie „Islam“ und „muslimische" Subjektivitäten in lokalen bzw. kommunalpolitischen Kontexten in Deutschland gouvernementalisiert, d.h., identifiziert, geformt und regierbar gemacht werden (Foucault, 2005). Damit liefert sie angesichts fortwährender Debatten um die Integration von „Islam“ in Deutschland und Europa einen wichtigen Beitrag zur Untersuchung ,,islambezogener" städtischer (Integrations-)Politiken und deren identitätspolitischen Effekten. Mehrere Studien unterstreichen die gegenwärtige Bedeutung eines politisch-administrativen Programms, das als Dialog mit dem Islam operiert und auf Integration durch Involvierung setzt (für die nationale Ebene: Tezcan, 2007; Amir-Moazami, 2011b; Dornhof, 2012). Der vorliegende Beitrag analysiert exemplarisch Praktiken lokaler Dialog-Maßnahmen und fragt nach deren Operationsweisen, Machteffekten sowie verknüpften Prozessen der Formierung von Subjekt- und Gruppenidentitäten. Er fasst lokale Dialogmaßnahmen als Machttechnologien, die auf die Re-Konfigurierung „muslimischer“ Identitäten zielen. Die lokale Perspektive verspricht dabei neue Einsichten, insofern der politische und gesellschaftliche Umgang mit „Islam“ bislang überwiegend mit Blick auf die natio- nale (und supra-nationale) Ebene thematisiert wurde (Koenig, 2003; Modood et al., 2006; Tezcan, 2009, 2012; Peter, 2008, 2010; Silvestri, 2010; Amir-Moazami, 2011a; Mavelli, 2013; Schiffauer, 2006; Schubert und Meyer, 2011; Isik und Schmitz, 2015). Ich werde argumentieren, dass der Dialog mit dem „Islam“ als eine emotionalisierte Technologie lokaler Vergemeinschaftung gefasst werden kann (De Wilde, 2015). Ich argumentiere, dass diese Technologie sowie die dadurch ermöglichten Normierungseffekte im Hinblick auf die Formung „muslimischer“ Identitäten auf ReKonfigurierungsprozessen des Verhältnisses zwischen Religion, (Lokal-)Politik und (Stadt-)Gesellschaft beruhen. Dazu greife ich die auch in der Geographie geführte Debatte um Post-Säkularität auf (Dressler und Mandair, 2011; Cloke und Beaumont, 2012), die den Blick auf die (Re-)Produktion des Verhältnisses zwischen säkular-staatlichen und religiösen Bereichen wie auch auf die dabei auftretenden Spannungen richtet.

Um Dialog-Politiken nach ihren Machteffekten und Subjektivierungsimpulsen befragen zu können, beziehe ich mich auf Michel Foucaults Studien zur Gouvernementalität. In diesen erarbeitete Foucault zunächst Perspektiven, um Transformationsprozesse von Staatlichkeit und staatlicher Macht(-ausübung) angemessen beschreiben zu können. 
Dabei konzeptionalisierte Foucault Formen gesellschaftlicher Herrschafts- und Machtverhältnisse als komplexe Verschränkungen von Führung und Selbstführung (Foucault, 2004 [1977-78), 2004 [1978-79], 1977, 2005). Seine Ideen wurden in den Sozialwissenschaften seither intensiv diskutiert und für machtanalytische Untersuchungen verschiedener Aspekte der (Selbst-)Steuerung von Gesellschaft eingesetzt (Rose, 1993, 2006; Burchell, 1993; Jessop, 2007; Bröckling und Krasmann, 2011; Ghatak und Abel, 2013; In der Geographie: Füller und Marquardt, 2009, 2010; Schreiber, 2011; Strüver, 2013; Marquardt, 2014, 2015). Foucaults anti-essentialistische Perspektiven auf Macht-Beziehungen (2005:254) ermöglichen es, lokale, situierte Praktiken der Machtausübung (als solche) zu rekonstruieren und in einen Kontext gesellschaftlicher Kräfteverhältnisse zu stellen (Jessop, 2007). Dabei lassen sich ,systematisch[e] Verbindungen zwischen Rationalitätsformen, Führungstechnologien und Selbstbezügen" (Bröckling und Krasmann, 2010:26) herausarbeiten. Dieser Ansatz fasst „Islam“ und „Muslime“ als Identitäten, die immer auch Resultat der Re-Konfigurierung gesellschaftlicher Kräfteverhältnisse (und daher in Anführungszeichen gesetzt) sind. Darauf aufbauend lässt sich fragen, wie „Islam“ und „Muslime“ zu Objekten lokaler Regierungspraktiken gemacht werden und welche Bedeutung dabei lokalen Kontexten und Entwicklungspfaden zukommt. Dem wird anhand einer ethnographischen Fallstudie in der Stadt Erlangen nachgegangen. Die Aufschlüsselung lokalräumlicher Aspekte von Regierungsprozessen stellt einen politisch-geographischen Beitrag zu gouvernementalitätstheoretischen Debatten dar, insofern letztere eine Sensibilität gegenüber der Vielgestaltigkeit lokaler Formen der Machtausübung propagieren (Bröckling und Krasman, 2010).

Diese Perspektiven führen in verschiedene Spannungsfelder hinein, die die gegenwärtigen Versuche städtischer Politiken prägen, kulturelle und religiöse Differenz unter liberalsäkularen Vorzeichen zu regieren. Im Kräftefeld des Dialogs werden bestimmte Vorstellungen von „Islam“ als wünschenswert artikuliert, diese sollen sich jedoch jenseits jeder Verordnung „von selbst“ aus den „muslimischen“ Gemeinden heraus entwickeln. Es geht vielfach um das Problem der Führung von als autonom konstituierten Subjekten/Gruppen, die sich selbst führen sollen (Rose, 1993). Der Beitrag wird Formen kommunalpolitischer Zugänge auf „Muslime“ als ein governing through friendship und damit als einen Modus von (Selbst-)Führung diskutieren, in welchem solche Widersprüchlichkeiten liberaler Regierungskunst (Foucault, 2005:180-185, 240-26; Burchell, 1993:267) spezifisch transformiert werden. So werde ich darstellen, wie in einem auf persönliche Begegnung, Informalität und Vertrauen setzenden Dialog eine „Atmosphäre der Behaglichkeit“ (De Wilde, 2015) praktiziert wird, die Führungsimpulse als „Ratschläge unter Freunden“ rahmt. Hierbei lässt sich auch diskutieren, inwiefern Emotionalität für Fragen gesellschaftlicher Steuerung bedeutsam werden kann (Tuori, 2009; Bar- getz und Sauer, 2010; D'Aoust, 2014), womit ein aktuelles Forschungsfeld der politischen Geographie aufgegriffen wird (Fortier, 2007; Schurr, 2013; Müller, 2015; Marquardt, 2015; Hutta, 2015).

Gleichzeitig wird illustriert, wie sich ein auf „persönliches Kennen(-lernen)" setzender Dialog als religionssensible Technologie ausgestaltet, die religiöse Empfindungen und Erfahrungen bearbeitbar werden lässt und sich in postsäkularen Spannungsfeldern zwischen religiösen Autonomien und säkular-staatlichen Gestaltungsmöglichkeiten konstituiert (Dressler und Mandair, 2011). Ich werde zeigen, wie der Dialog durch spezifisch sensibilisierte, post-säkulare Experten/innen vermittelt wird, die als religiös bewegte Subjekte mit lokalpolitischer und/oder wissenschaftlicher Erfahrung Prozesse eines „Religion-Making“ (ebd.) moderieren. Mit Blick auf die den ,empirischen Gegenstand“ durchziehenden „Maschen der Macht“ (Foucault, 2005:239) lassen sich diese Aspekte mit den Überlegungen zu liberalen Führungstechniken verknüpfen.

Die nachfolgenden Kapitel diskutieren zunächst Dynamiken der Problematisierung von „Islam“ in Deutschland, die mit kommunalpolitischen Formungsprozessen verschnitten sind. Anschließend werden lokale „Dialog“-Praktiken exemplarisch illustriert und hinsichtlich der skizzierten Fragen interpretiert.

\section{Der (interreligiöse) Dialog mit dem „Islam“}

Verstärkt seit den 2000er Jahren sind „Islam“ und „Muslime" zu den Objekten integrations- und sicherheitspolitischer Überlegungen und Maßnahmen in Deutschland und Europa geworden (Schiffauer, 2006, 2008; Tezcan, 2007; Silvestri, 2010; Rodatz, 2012; Mavelli, 2013; Spielhaus, 2013). Diese Zugriffe reproduzieren vielfach die Vorstellung einer homogenen, spezifisch integrationsbedürftigen ,muslimischen“ Bevölkerungsgruppe und können ferner als Ausdruck einer allgemeinen Kulturalisierung gesellschaftlicher Selbstbeschreibungen gedeutet werden (Tezcan, 2011b). Integrationspolitiken in Deutschland setzen dabei verstärkt auf einen (interkulturellen/-religiösen) Dialog mit kulturell definierten Gruppen (Tezcan, 2006, 2012). Dem Dialog mit dem „Islam“ kommt hierbei eine große Bedeutung zu, was sich in der Etablierung der Deutschen-Islam-Konferenz im Jahre 2006 widerspiegelt, die auf die Institutionalisierung eines „Dialog[s] zwischen Staat, Muslimen und Gesellschaft“ (damaliger Innenminister Schäuble, DIK, 2009:2) zielt.

Während religiöse Identitäten im „Dialog der Kulturen“ grundsätzlich an Relevanz gewannen (Tezcan, 2006:1), werden verstärkt auch interreligiöse Dialoge integrations- und sicherheitspolitisch aufgegriffen (Tezcan, 2007; Dornhof, 2012; Klinkhammer et al., 2011). Interreligiöse Dialoge können hierbei als eine Regierungstechnik im Hinblick auf die Integration von „Islam“ gedeutet werden, die bspw. „,christliche“ Institutionen als ,gesellschaftliche Platzanwei- 
ser“ (Rommelspacher, 2012:218) für den „Islam“ positioniert. Gerade der christlich-islamische Dialog ,verlässt damit den ursprünglichen, exklusiven Ort der religiösen Gruppen und wird [...] zu einem politischen Programm" (Tezcan, 2009:67), in dessen Kräftefeld Subjektivitäten verhandelt werden (Ami-Moazami, 2011b). Bisweilen wird gar der gesamte gesellschaftliche Umgang mit „Islam“ als (partiell) ,interreligiöser Dialog“ "gerahmt (Tezcan, 2007; Dornhof, 2012), wodurch integrationspolitische Problemstellungen als ,islamische“ Probleme reproduziert werden können.

Eigene Erhebungen zu Integrationspolitiken in deutschen Großstädten (2015) ${ }^{1}$ zeigen, dass auch auf städtischer Ebene vielfach Maßnahmen ausgestaltet werden, um lokale ,muslimische" Gemeinden in einen partnerschaftlichen Dialog zu involvieren. In Anlehnung an Rose (1996) kann dieser kommunale Dialog als ein Regieren durch community gefasst werden. Einerseits wird die Stärkung interkulturell und -religiös gerahmter Begegnungen vor Ort zum Programm. Ferner geht es dezidiert um die Mobilisierung der Selbstführungskompetenzen „muslimischer“ Gemeinden (Tezcan, 2007). Aus dieser Perspektive adressieren Stadtpolitiken die „muslimische“ Gemeinschaft als einen lokalen Nährboden für die Herausbildung eines bestimmten „muslimischen“ Subjekts: Gemäß meiner Untersuchungen in Erlangen wäre es das dialogbereite, (theologisch) selbst-reflexive, säkulare, sozial-politisch engagierte und transparente „muslimische“ Subjekt, welches sich innerhalb einer spezifisch vernetzten „muslimischen“ Gemeinschaft formen möge. Jener „,[sich] seiner selbst bewusste Muslim“ (Tezcan, 2007:68) wird dabei auch ,in der Schmiede des interreligiösen Dialogs geformt, bevor er als ethisch/politisch verantwortbarer Kulturvertreter die politische Bühne betritt" (ebd.: 68).

Viele Städte nutzen interreligiöse Dialogforen, um einen vertrauensbasierten Zugang zum lokalen „Islam“ zu etablieren. Dies mag auch damit zu tun haben, dass der Umgang mit Religionsgemeinschaften allgemein als emotionales Terrain aufgefasst wird. So stellen die vom bundesweit operierenden „Kommunalen Qualitätszirkel zur Integrationspolitik“ veröffentlichten Empfehlungen zum Umgang mit religiöser Vielfalt diesen als ,eine große Herausforderung " für die Kommunen dar, da ,gerade beim Thema „Religion“ [...] alle Beteiligten auch emotional berührt [sind]“ (KQI, 2012:21). Entsprechend könne die kommunalpolitische Bearbeitung von Religion, so eine Handreichung des „Netzwerk-IntegrationBayern“, „nicht von jedem“ (Kuhla und Szukitsch, 2011:14) erfolgen und müsse auf ,persönliche[r] Kontaktaufnahme und -pflege“ sowie „Vertrauensbeziehung[en]“ (ebd.: 16) basieren. Beispielsweise lagerte die Stadt Erlangen Maßnah-

${ }^{1} 2015$ führte der Beitragsverfasser zusammen mit Prof. Georg Glasze und Studierenden des Masterstudiengangs „Kulturgeographie“ am geographischen Institut der Universität ErlangenNürnberg eine Studie durch, in der Integrationsbeauftragte aus 52 deutschen Großstädten zur Ausgestaltung kommunaler islambezogener Politiken befragt wurden. men zur Vernetzung „muslimischer“ Gemeinden mit städtischen Sozialberatungsangeboten an zivilgesellschaftliche Projekte aus, deren „,muslimisch“-gläubigen „ProjektmitarbeiterInnen $[\ldots]$ es aufgrund $[. .$.$] ihres sozialen und religi-$ ösen Hintergrundes möglich [sei], authentisch und vertrauensvoll in den islamischen Gemeinden zu arbeiten" (Stadt Erlangen, 2009:3-4). Vielfach wird auf „Vertrauensarbeit mit den Moscheen" (Integrationsbeauftragter 1, eigene Studie) gesetzt. Hierbei werden interreligiöse Dialogforen als Schutzräume bedeutsam, ,in [denen] das Wissen voneinander und Manieren der wechselseitigen Behandlung ausprobiert werden“ (Tezcan, 2009:67; Klinkhammer et al., 2011), sowie zu Instrumenten, durch die man ,eine Vertrauensbasis schafft, [um] auch durchaus schwierige Themen ansprechen [zu] können“" (Integrationsbeauftragter 2).

\section{Der „Erlanger Dialog“: Methodik, Feldzugang und Feldcharakteristika}

Die Empirie für diesen Beitrag basiert auf einem ethnographischen Zugang zu den Orten und Praktiken der lokalpolitischen Verhandlung von „Islam“ und „Muslimen“ in der Stadt Erlangen (teilnehmende Beobachtungen, informelle Gespräche sowie Einzel- und Gruppeninterviews). ${ }^{2} \mathrm{Um}$ die Regierbarmachung von „Islam“ zu fassen, wurde mit einer praxisanalytischen Ethnographie gearbeitet, die auf die Rekonstruktion von Praktiken der Machtausübung im Vollzug abzielt (Wrana und Ott, 2010; Müller, 2012). Ziel war es herausarbeiten, welche Funktion bestimmte Praktiken im Kontext der Normalisierung und Rationalisierung von Identität(en) und Subjektivierungsformen aufweisen und inwiefern sie damit als Regierungspraktiken wirken können. Insgesamt behandelt der Beitrag nicht alle Themenfelder und Maßnahmen des kommunalen Umgangs mit „Islam“, sondern analysiert mit Blick auf eine „Mikrophysik der Macht im Gesprächskreis“ (Schiffauer, 2008:218) vorwiegend situierte Aushandlungsprozesse in städtischen Dialoginitiativen.

Dabei erschienen mir diese Aushandlungsprozesse zunehmend als eine gewissermaßen emotionalisierte politische Praxis (vgl. Kap. 4). Hierbei ist Emotionalität/Emotionalisierung als ein Phänomen zu konzeptionalisieren, das in diskursiv konstituierten Aushandlungssituationen als solches erst hergestellt, mit körperlichen Ausdrucksformen verknüpft, sichtbar, greifbar sowie mitunter zum Element von Machtausübung gemacht wird (Marquardt, 2015).

\footnotetext{
${ }^{2}$ Ich führte Interviews mit Vertretern/innen lokaler Politik und Verwaltung, der lokalen politischen Parteien, der „muslimischen" Gemeinden und Organisationen (mit Gemeindemitgliedern, Vorstandsmitgliedern und Imamen), mit Vertretern/innen interreligiöser Dialoginitiativen, der ,christlichen“ Kirchen und ihrer lokalen Bildungswerke sowie der Universität. Ich besuchte zwischen 2014 und 2016 zahlreiche Sitzungen der „ChristlichIslamischen-Arbeitsgemeinschaft" und des stadtpolitischen Initiativkreises „Freundeskreis-muslimischer-Gemeinden-in-Erlangen“, lokale Veranstaltungen und Ausstellungen
} 
Unter der Annahme, dass Emotionalität vielfach über körperliche Ausdrucksformen eine unter bestimmten diskursiven Bedingungen plausible Repräsentanz erhält, wurden folglich nicht-sprachliche Aspekte besonders beachtet. Körperbewegungen, mimische, gestische und akustische Aspekte (z.B. Intonationsweisen) sowie das räumlich-körperliche Arrangement der Dialogpraxis fanden in die Beobachtungsprotokolle Einzug. So wurde Emotionalität als Attribut und Effekt situierter Interaktions-Arrangements fassbar, die je eigene Atmosphären generieren (Michels, 2015).

Die Praxis des Dialogs in Erlangen weist eine interessante Doppelstruktur auf. Bereits 1996 gründete der damalige Oberbürgermeister die „Christlich-IslamischeArbeitsgemeinschaft" (CIAG). Das Forum wurde als interreligiöser Dialog ausgerichtet, doch trotz dieser Programmatik seit Anbeginn aktiv von der Stadt selbst ko-moderiert und gestaltet. So werden de facto überwiegend integrationspolitische Themen hinsichtlich der Involvierung einer ,,muslimischen" Bevölkerungsgruppe verhandelt. Diese integrationspolitische Überformung des interreligiösen Dialoges ist ein überlokales Phänomen (Tezcan, 2006; Klinkhammer et al., 2011). Trotzdem finden hier theologische Diskussionen auch weiterhin statt. Moderiert wird die CIAG von einer „muslimischen" Sprecherin (Konvertitin, kommunalpolitisch aktiv) und einem ,christlichen“ Sprecher. Letzterer ist eine kommunalpolitische Koryphäe, im Stadtrat und in kommunalen Ausschüssen aktiv, sowie Religionsbeauftragter der SPDStadtratsfraktion.

Zudem gründete die Stadt 2010 den „Freundeskreismuslimischer-Gemeinden-Erlangen e.V.“ (FMGE). Im Gegensatz zur CIAG geht es hier auch programmatisch primär um die Unterstützung lokaler „,muslimischer“ Gemeinden im Sinne einer proaktiven städtischen Integrationspolitik. $^{3}$ Moderiert wird der FMGE von ebenjenem SPD„Religionsbeauftragten“, der zweiten Bürgermeisterin sowie einem ehemaligen Vorsitzenden des Ausländer- und Integrationsbeirats (allesamt ,nicht-muslimisch"). In beiden Foren sind Repräsentanten/innen der Stadt, der „,christlichen“ Kirchen und Bildungswerke, der „muslimischen“ Gemeinden (die 2000 einen Dachverband gründeten) und der Universität vertreten, dazu einzelne ,muslimische“ und ,christliche“ $\mathrm{Ge}$ meindemitglieder sowie Einzelpersonen aus Politik, Stadtgesellschaft und Wirtschaft. Auch die zweite Bürgermeisterin nimmt regelmäßig an beiden Dialog-Initiativen teil. Mitunter aufgrund zahlreicher personaler Überschneidungen über-

\footnotetext{
${ }^{3}$ Der FMGE gründete sich, als die „Islamische-GemeindeErlangen“ im Bericht des bayerischen Verfassungsschutzes auftauchte. Diese Beobachtungsentscheidung wurde von Erlanger Kommunalpolitikern/innen und Kirchenvertretern/innen kritisiert, worauf man einen Initiativkreis gründete (Interviews mit Erlanger Vertretern/innen aus Politik und Verwaltung, 2014-16; http://www.nordbayern.de/region/erlangen/ freundeskreis-der-muslime-sucht-halt-in-paragraphen-1.787827, 13.07.2017).
}

kreuzen sich die programmatischen Ausrichtungen der Initiativen (interreligiöser und integrationspolitischer Dialog).

Diese Doppelstruktur und die inhaltlichen Überkreuzungen werden interessanterweise besonders gutgeheißen. Ein anerkennender und tiefgehender Dialog mit den ,muslimischen" Gemeinden, so die Logik, müsse auch für Fragen von Religion/Glauben offen sein. Diese Erkenntnis habe sich in Erlangen nach anfänglicher Skepsis gegenüber dem Thema „Religion“ im Laufe der 2000er Jahre allmählich durchgesetzt (Interviews mit Vertreter/innen lokaler Politik und Verwaltung, 2015-16). Nun werde begrüßt, dass einzelne, (inter)-religiös interessierte städtische Vertreter/innen als „Schaltstellen“ (Selbstbezeichnung des SPD-,,Religionsbeauftragten") zwischen Stadt, Kirchen und Moschee-Gemeinden agieren, über informell-persönliche Kontakte und Netzwerke Vertrauensarbeit leisten sowie „Islam“-bezogenes Wissen aufbauen (Sorgen um religiösen Extremismus scheinen dabei solche Maßnahmen mitzutragen). Insgesamt profiliert sich der Dialog (nicht nur) in Erlangen über das Versprechen, in Abgrenzung zu den als vorurteilsbeladen aufgefassten öffentlichen Diskussionen um „Islam“ eine auf „Verständnis“, „Augenhöhe“ und „Vertrauen“ basierende, pragmatische Involvierung von „Muslimen“ praktizieren zu können (Erhebungen 2014-16; Klinkhammer et al., 2011).

Ich möchte nun in die Analyse ausgewählter Sitzungen der Dialogprojekte CIAG und FMGE überleiten. Ich skizziere, wie ein auf „Dialog“ setzender Zugang zu „muslimischen“ communities sich lokal ausgestaltet und wie dabei religiöse Identitäten im Modus einer Gouvernementalität der Freundschaft (neu) ausgerichtet werden.

\section{Gouvernementalität der Freundschaft: Schlaglichter}

\section{1 „Unter Freunden ... .}

Der Dialog mit dem „Islam“ in Erlangen ist von einer auffälligen Informalität geprägt. Alle Teilnehmer/innen sind um eine lockere Umgangsweise bemüht. Diese begründet eine emotionalisierte Atmosphäre des Miteinanders, die als Element einer politischen Praxis zu denken ist, welche auf kontinuierliche persönliche Bekanntschaften setzt (Fortier, 2007; De Wilde, 2015). Entsprechend duzt man sich, scherzt miteinander und lacht, erzählt persönliche Geschichten, umarmt sich und pflegt eine nicht-hierarchische Sitzordnung. Diese Praktiken nehmen in den Sitzungen viel Zeit in Anspruch, haben rituellen Charakter und eine affizierende Wirkung. Sie werden auch programmatisch als Besonderheit „begegnungsorientierter" Kommunalpolitik artikuliert. Hierbei partizipieren städtische Vertreter/innen aktiv am Gespräch religiös bewegter Menschen.

In einer der städtisch moderierten Sitzungen der CIAG wurde bspw. das Thema „Islam und religiöse Gewalt“ behandelt. Ermuntert durch den „christlichen Sprecher“ - gleich- 
zeitig städtischer Repräsentant - formulierte der Direktor der lokalen katholischen Erwachsenenbildung eine zugespitzte Kritik am „Islam“: Er erachte das „christliche“ Motiv der Nächstenliebe als politisch sinnvoller, da friedensethisch radikaler, als ableitbare ,islamische“ Friedensbotschaften. Es war ihm allerdings sichtlich unangenehm, diese Kritik zu formulieren. Es doch zu tun ermöglichte ihm der Verweis, dass man „hier doch im Vertrauen und unter Freunden sei““ und „ehrlich miteinander reden könne“ (Zitate, Beobachtung 2015). Er legitimierte seine Kritik mit einem institutionalisierten Freundschaftsmotiv - und dies erfolgreich. Er erschien als ein offen/ehrlich sprechender Verbündeter, der zwar kritisiert, aber selber ,leidet“, weil er nicht verletzen will. Dieses „Hin-und-her-gerissen-sein“ fand körperlichen Ausdruck (z.B. beschwichtigende Gesten und Intonation, verständnissuchende und -volle Blicke). Entsprechend schlossen an diese Szene Praktiken der Vergemeinschaftung an. Die ,muslimischen“ Vertreter/innen bestätigten, dass man im Dialog alles sagen dürfe und es wurde vereinbart, dass der „Kritiker“ in der nächsten Sitzung ein Referat halten könne. Wohlplatzierte Witze lockerten die Stimmung - ein „,muslimischer“ Gemeindevertreter fragte, ob die kritischen Fragen an den ,Islam“ dann ,,auswärts oder als Heimspiel“" organisiert werden - gemeint war, ob in einer Moschee oder anderswo. Es wurde gelacht. Der städtische/,,christliche“ Moderator lobte die Runde für das vertrauliche und offene Miteinander. Der Konflikt schien innerhalb dieser Vergemeinschaftungsrituale als abgepuffert. Bedeutsam für die Aushandlungsdynamiken war hierbei eine - in die Praxis des Dialogs eingebettete - Sichtbarwerdung emotionalisierter Körper, über die die Gesamtsituation als eine ,ehrlich-authentische“ ausgehandelt werden konnte. Innerhalb diskursiv konstituierter Situationen wird Emotionalität als Teilelement sozialer Praxis bedeutsam, das in bestimmten doings und sayings (Schatzki, 2002:71) Repräsentanz erhält. Das Beispiel zeigt zudem ein symptomatisches Kippbild, insofern durch den Verweis auf „Freundschaft“ eine bestimmte Kritik ermöglicht, andererseits aber Gegenkritik erschwert wird. Das Freundschaftsmotiv produziert eine emotional aufgeladene Verpflichtung für die anwesenden „Muslime“, die Kritik „eines Freundes“ nun auch anzunehmen. Grundsätzlich scheinen städtische Politiken daran interessiert, dass gewisse Dialog-Teilnehmer/innen (z.B. „Christen“) unter städtischer Moderation kritische Fragen an den „Islam“ stellen. Andererseits erhält die ,muslimische“ Seite durch das Freundschaftsparadigma eine gewichtige Sprecherposition und kann ebenso unter Rekurs auf ,gegenseitiges Vertrauen“ operieren.

In der folgenden Sitzung der CIAG hielten der Vertreter von „Bildung-Katholisch“ und ein eingeladener ,,islamischer" Theologe vom 2012 in Erlangen gegründeten Islamisch-Theologischen Institut jeweils ein Referat. Insgesamt wurde hierbei ein bestimmtes Islambild forciert, das eines dialogbereiten, offenen, auf möglichst allgemeinen ethischen Prinzipien ruhenden „Islam“, sowie damit zusammen- hängend die Idee einer individuellen und verinnerlichten ethisch-religiösen Selbsthaltung des „muslimischen“ Subjekts. Demgegenüber wurde die Vorstellung eines verrechtlichten, normativistischen und strikt-handlungsanweisenden „Islam“ kritisiert. Der ,islamische“ Theologe gab hierfür Impulse, Vertreter/innen der Stadt, der ,,christlichen“ Kirchen und Bildungswerke als auch einige „muslimische“ Sprecher/innen nahmen diese auf. Wieder andere ,muslimische" Gemeindemitglieder wirkten deutlich reservierter. Hinsichtlich einer vermeintlichen ,Verwissenschaftlichung““ des „Islam“ wird das Islamisch-Theologische-Institut bisweilen auch kritisch betrachtet. Neben ,,christlichen“ Vertreter/innen bezogen sich auch städtische Repräsentanten/innen explizit auf islamisch-theologische und exegetische Motive: So das Motiv des „Islam“ als Baum mit vernachlässigbaren Zweigen (Traditionen) und einem universal-humanistisch ausgerichteten Kern/Stamm, dessen allgemein-ethische Prämissen „Islam“ als rituelle Praxis transzendieren und qua dieser Verallgemeinerung das „,islamische Subjekt“ zum Dialog mit „,nicht-islamischen“ Positionen befähigen. Solche Motive wurden dann mit Begriffen wie ,erhellend“ oder ,,inspirierend“ markiert. Auch traditionell-,,islamische“ Exegese-Konzepte wie itihād (arabisch: „Anstrengung“) und fikr (,Denken“) wurden von ,muslimischen“ und ,nichtmuslimischen“ Dialog-Teilnehmern mobilisiert, um in Hinblick auf religiöse Wahrheitsvorstellungen kritisches Denken und (Re-)Interpretieren, Interpretationsvielfalt und die Bedeutung der Vernunft zu untermauern (zu itihād vgl. Rochdi (2008), selbst sporadischer CIAG-Teilnehmer). ${ }^{4}$

\subsection{Positive Orts-Energien!?}

Die Bürgermeisterin begann daraufhin eine emotionale Rede, in der sie betonte, wie wertvoll es sei, dass solche Visionen eines ethisch-dialogischen ,Islam“ ,hier in Erlangen, in unserer Stadt“ (Originalzitat) entstehen. Dies zeige das vertrauensvolle und freundschaftliche Verhältnis, welches offene Diskussionen zwischen „Muslimen“ und „Nicht-Muslimen“ ermögliche. Diese Praktik des „Lobens“ appelliert an die Emotionalität des Vertrauensmotivs, konstituiert die Anwesenden als eine Gemeinschaft und den ,runden Tisch“ als Ort positiver Energien. Mit dem Ziel der Begeisterung für das gemeinsame Projekt wird eine ,emotionale Disposition“ (Marquardt, 2015:176) (der Zustimmung) forciert. Die Motive des Vertrauens und der Freundschaft werden sodann über das Rekurrieren auf die städtisch-lokale Identität verständlich gemacht. Die Stadt Erlangen wird als der Ort artikuliert, an welchem Vertrauen gedeihen konnte und Begegnung möglich wird. Hier zeigt sich, wie ,,affective intensities are at play that allow one to nest within [or] open up to [...] spaces" (Hutta, 2015:301). Die Bürgermeisterin und die ,muslimi-

\footnotetext{
${ }^{4}$ Auch Abu Zayds Artikulation des Koran als Gottes Menschenwort - und damit als kritisierbar - wurde thematisiert (dazu: Mahmoud, 2006).
} 
sche“ Sprecherin lobten gleichzeitig die anwesenden „,muslimischen" Gemeindevertreter/innen für ihre Bereitschaft zur kritischen Reflexion religiöser Fragen und knüpften solche Haltungen an die Zukunftsvision einer progressiven, offenen, für alle vorteilhaften Stadtgesellschaft. Die (,,christlichen“ und „muslimischen“) ModeratorInnen der CIAG, die Bürgermeisterin und der ,islamische“ Theologe agieren hier in Allianz als post-säkulare Experten/innen (Vgl. 5.2). Als solche sind sie an der integrativen Behandlung kommunalpolitischer und theologischer Fragen interessiert. Der „Dialog unter Freunden" wird dabei zum konstant heraufbeschworenen Imaginär, welches Führungs- und Formungsansprüche absorbiert und als wohlmeinende Ratschläge rahmt.

Die Lobrede der Bürgermeisterin erscheint somit auch als eine Technik der Ordnungsherstellung sowie mitunter als ein paternalistisches Moment, welches die anwesenden „Muslime" auf emotionaler Schiene dazu drängt, sich kritisch mit ihrer Religion auseinanderzusetzen (wobei gerade die Emotionalisierung Widerstände erschwert). Gleichwohl aber erscheint die Rede auch als ein Beispiel für die stete Arbeit am gemeinsam geteilten Mythos von Vertrauen und Freundschaft, das von allen Parteien, und besonders auch auf ,,muslimischer" Seite, durchaus als bewegend und ermächtigend erfahren wird. Wie also sind diese Momente zu interpretieren? Handelt es sich hier um eine konstruktive, wahrhaft agonistische (Mouffe, 2007) und emanzipative Auseinandersetzung, die gerade aufgrund der praktizierten Emotionalität Formen authentischer Rede freilassen kann? Oder handelt es sich um eine Post-Politik (Ranciere, 1995; Crouch, 2004), in der Aushandlungsprozesse weniger von Kontroverse als von einem Formalismus beschworener Gefühle getragen werden? Eine Politik, die ,im Namen des Dialogs“ und durch Techniken der Emotionalisierung Konsens schafft, wo keiner ist und Führungsansprüche verschleiert? Ich denke, es ist beides, was governing through friendship ausmacht. ${ }^{5}$

Der Dialog setzt vielfach auf die Produktion greifbarer Erfahrungen gemeinschaftlichen Zusammenhalts. Als Vergemeinschaftungstechnologie operiert er durch affektive Teilhabe (affective citizipenship; vgl. De Wilde, 2015) und ruft das Subjekt zur emotionalen (An-)Bindung an die Dialoggemeinschaft sowie an deren gemeinsame, lokal-städtische Lebenswelt auf. Ein Effekt ist die Modellierung durchaus (handlungs-)ermächtigender Gefühlsintensitäten. Mehrere „muslimische“ Gemeindemitglieder erwähnten, dass sie sich ,im Verlauf der Jahre auf den Straßen zunehmend sicherer fühlen würden" und knüpften diese Erfahrungen an die Existenz des Dialogs (Beobachtungen, 2015). Ein Moschee-Vorstandsprecher erwähnte, dass er sich ,in einer Stadt selbstbewusster fühle“ in der es ,einen [betont:] Freundeskreis der Muslime" gibt. Ohne in diesem Beitrag eine Ontologie des Affekts leisten zu können, möchte ich mit Camp-

\footnotetext{
5 „Techniken“ repräsentieren hierbei keineswegs intentionale Strategien, sondern etablierte/normalisierte Verfahrensweisen sozialer Interaktion.
}

bell (2010) andenken, dass Regierungstechnologien spezifische „Emotionalitäten des Regierens“ inhärent sein können, die als modellierte (Gefühls-)Intensitäten Subjektivierungsprozesse kanalisieren.

\section{3 „Ruhig etwas rumspinnen“}

In Erlangen wird derzeit der bundesweit erstmalige Versuch unternommen, eine „muslimische“ Erwachsenenbildung „Bildung-Muslimisch“ nach „,christlichem“ Vorbild zu etablieren. Diesem Projekt widmet sich der „Freundeskreis“ FMGE. Während viele Vertreter/innen der „muslimischen“ Gemeinden in einer Erwachsenenbildung primär eine „Aufklärung" der Mehrheitsgesellschaft über den (richtigen) „Islam“ erkennen, sehen die Stadt sowie andere „muslimische" Vertreter/innen darin auch ein Mittel, einen selbstreflexiven und selbstkritischen „Islam“ zu fördern. Dafür möchte man u.a. (nicht-organisierte) ,islamische“ Positionen/Stimmen involvieren, die bisher im Dialoggeschehen nicht repräsentiert waren. Ziel sei dann ein lokales Forum, in dem sich verschiedenste ,islamische“ und andere weltanschauliche Perspektiven gemeinsam mit der Stadtgesellschaft austauschen und darüber ihre Dialogfähigkeit einüben. Die Stadt hat hierzu Vertreter/innen der evangelischen Erwachsenenbildung gebeten, den „muslimischen“ Gemeinden zu zeigen, wie die ,christlichen“ Kirchen Erwachsenenbildung organisieren. Die Botschaft war: Um „Bildung“ und „kritischen Diskurs“ zu ermöglichen, müsse man aus den religiösen Gemeinden heraustreten, eine Reibungsfläche zum traditionellen „Kirch(Moschee-)gängertum“ etablieren: „Bloß nicht nachplappern, was der Pfarrer sagt“, wie die Bildung-Evangelisch-Vertreterin betonte (Protokoll 2015). Man empfahl den „muslimischen“ Gemeinden, in den Organisationsausschuss der Erwachsenenbildung auch „Nicht-Muslime“ sowie „Moschee-kritische-Muslime“ zu involvieren und keinesfalls vorab Glaubensperspektiven zu exkludieren. Hierbei nickte die eingeladene Professorin des Islamisch-theologischen Instituts bekräftigend.

Das Treffen fand in einer Moschee statt. Als der Gebetsruf ertönte, wurde die Sitzung unterbrochen und die ,muslimischen" Teilnehmenden brachten sich ein Stockwerk tiefer zum Beten ein. In dieser Situation fragte der Moderator des Freundeskreises (sicherlich der profilierteste „AgendaSetzer“): „Sagt mal, meint ihr, wir drücken denen hier was auf?" Es kam gar zu einer Diskussion darüber, wie man Vorschläge geben könne, ohne ,aufzudrücken“. Die Fortführung der Sitzung wurde dann von einer selbstreflexiven Rede eingeleitet. Der Moderator sagte: „Hört mal, wir wollen euch nichts aufdrücken. Wir zeigen euch, wie es gehen könnte, aber das ist nicht die einzige Möglichkeit. Vermittelt bitte den Gemeinden, dass wir uns aus tiefstem Herzen wünschen, dass hier etwas entsteht, das [...] euch [Muslime] in eurem Selbstverständnis und -bewusstsein stärkt [...]. Nehmt euch Zeit, darüber nachzudenken, ob ihr das so wollt". Diese emotionale Rede wurde entsprechend angenommen: „Das wis- 
sen wir doch, du drückst uns nichts auf" war die Antwort eines Moscheeverein-Vertreters, mit versöhnlicher Intonation, woraufhin der städtische Repräsentant erwiderte: „Das ist nett, aber wirklich, richtet aus, wir wollen nichts aufdrücken“ (Protokoll 2015).

Ich deute diese Praktiken als ein governing through friendship: Eine Technologie der (Selbst-)Führung, die in verhältnismäßig selbstreflexiven und sich selbst relativierenden Formen politischer Einflussnahme ihren Ausdruck findet. Dabei liegt jener Praxis der Selbstreflexivität selbst eine einflussnehmende, formende Kraft inne, gerade weil sie am emotional aufgeladenen Vertrauensmotiv ansetzt und an diesem arbeitet. In dieses Motiv wird von allen Seiten investiert, und dazu gehört die beständige Metareflexion des ,guten“ Umgangs miteinander, eine Form institutionalisierter Sorge. Im Vollzug solcher Techniken konstituieren sich die DialogTeilnehmenden performativ als Vertraute und Ratgeber, die es mit ihrem Gegenüber gut meinen. Diese - das Subjekt qua Gemeinschaftserfahrung ermächtigende - Art der Beratung erhält etwas Persönliches, bisweilen auch Paternalistisches.

Nachfolgend wurde die Idee, in den Trägerverein einer „muslimischen“ Erwachsenenbildung „,nicht-muslimisches“ Personal zu integrieren, vom städtischen Moderator unter Bezugnahme auf seine eigenen Erfahrungen als „Katholik“ begründet. Als ,Katholik “ partizipiere er im Kuratorium von „Bildung-Evangelisch“ und habe hierbei die Erfahrung gemacht, dass es ,erfrischend und spannend“ sei, ,einmal eine Außenperspektive auf den eigenen Glauben zu erleben“ (Protokoll 2016). Dies würde einen selbst in der religiösen Entwicklung weiterbringen und die eigene Glaubensperspektive letztlich festigen. Überhaupt würde sich eine Religionsgemeinschaft, die ihre Glaubensansichten kritisch reflektiert, gesellschaftlich besser integrieren können. Dies wünsche man den „muslimischen“ Gemeinden. Die Sprecherin von Bildung-Evangelisch, Diplom-Religionspädagogin und mit der akademischen Theologie vernetzt, gab diesen Argumenten mit ihrer Präsenz eine religionspädagogischwissenschaftliche Rahmung. In einem elanvollen Statement legte sie den ,muslimischen“ Gemeindevertretern/innen nahe, bei der Themenfindung für ein religiöses Bildungsprogramm kreativ zu sein und „ruhig etwas rumzuspinnen“, insofern „das Diskutieren verschiedener Glaubensansichten ja auch Spaß machen kann“ (Protokoll 2016). Einige „muslimische" Gemeindevertreter/innen griffen diese Argumentationen auf. Nachdem ein „muslimischer“ Vertreter anmerkte, dass viele „Muslime“ in den Gemeinden allzu dogmatisch der Ansicht seien, im Koran stehe bereits alles (zur Lebensführung Relevante) geschrieben, wurde dies als problematisches Schriftverständnis diskutiert, welches bildungspolitisch anzugehen sei. Zusammenfassend praktizieren religionssensible Experten/innen - die sich in kooperativen Arrangements quer zur Einteilung ,muslimisch/nichtmuslimisch" formieren - den Versuch, bestimmte Formen religiösen Selbstverständnisses im „,muslimischen“ Gemeindeumfeld zu implementieren. Dabei wird auf die Selbststeue- rung des Subjekts durch die Internalisierung bestimmter Haltungen/Selbstverständnisse abgezielt. Gleichzeitig umfassen solche „Prozesse der Subjektivierung [immer auch] Versuche der Erzeugung und Justierung von Gefühlen“ (Marquardt, 2015:176): Selbstreflexion fühle sich gut an.

Bezüglich „Bildung-Muslimisch“ wurde letztlich ein Kompromiss ausgehandelt. Gemäß den städtischen Vertretern/innen würde das Projekt nur über die Involvierung möglichst vielfältiger Positionen gesamtgesellschaftlichen Rückhalt genießen. Einige „muslimische“ MoscheegemeindeVertreter/innen bejahten dies, während andere - ebenfalls mit dem Vertrauensmotiv operierend - mahnend anfügten, dass das Projekt vollständig gemeindegetragen sein müsse, da es um die Sicherstellung einer ,street-credibilty innerhalb der Gemeinden“" gehe (Zitat, 2016). Die MoscheeGemeinden würden zudem den „normalen Islam“ darstellen und hätten ihre Kooperationsfähigkeit vielfach bewiesen. Die Plausibilität dieser Argumente stellt einen Effekt des Dialogs dar, insofern die Gemeinden jahrelang als legitime „Islam"-Vertreter konstituiert wurden. Sodann wurde festgehalten, dass der Trägerverein des Projekts „100\% muslimisch“ (Original-Zitat!) sowie gemeindegetragen sein müsse, sich um diesen herum aber zwingend ein beratendes Kuratorium bilden werde, das auch anderweitige (nicht„muslimische“, „Moschee-ferne“) Perspektiven integrieren soll. Diese Diskussionen vermeintlich rein organisatorischer Aspekte religiöser Bildungsinfrastrukturen stellen (trotz gelegentlicher Bekräftigungen säkularer Trennungsprinzipen) Formen politischer Einflussnahme auf Glaubensverständnisse dar.

\section{Gouvernementalität der Freundschaft: Kontexte}

\subsection{Post-Säkulare Perspektiven}

Im Dialog wird das selbst-reflexive/selbst-kritische religiöse Subjekt geformt. Dieses soll durch Hinterfragung eigener Dogmen eine gegenüber religiöser Wahrheit distanzierte Haltung entwickeln, dadurch Religion keinesfalls zur Grenze in sozialen Interaktionen werden lassen, aus eigenem Religionsverständnis heraus eine religionsneutrale, tolerante Ethik internalisieren und folglich „günstig“ mit säkularistischen Ordnungsprinzipien zusammenfallen (Mahmoud, 2006; Zur Verknüpfung von Selbstreflexivität und Säkularismus: Mandair und Dressler, 2011). ${ }^{6}$ Das Subjekt soll diese Haltung theologisch begründen und praktisch einüben (z.B. in der Erwachsenenbildung). In Anlehnung an Mahmoud kann hier von der Reorganisation religiöser Subjektivitäten nach Maßgabe einer „liberal political rule“ (Mahmoud, 2006:328) gesprochen werden, die als Produktion des säkularen Subjekts operiert. Dieses Projekt gründet sich „not so much in the se-

\footnotetext{
${ }^{6}$ Säkularismus bezeichnet hier einen politisch-philosophischnormativen Diskurs, der die Trennung von Religion und Politik spezifisch plausibilisiert (Mahmoud, 2006).
} 
paration of state and religion [...] but in the kind of subjectivity that a secular culture authorizes [...]" (ebd.: 328; zur Kategorie „säkularer Muslime“: Tezcan, 2011a; AmirMoazami, 2011a; Mavelli, 2013; Schiffauer, 1997).

Derartige Analysen der politischen Bearbeitung religiöser Selbstverständnisse im säkular-staatlichen Kontext konzeptionalisieren das „Säkulare“ und das „Religiöse“ nicht als gegebene/vorab-existente „Bereiche“, sondern als (epistemologische) Kategorien, die fortwährend in gegenseitiger $\mathrm{Ab}$ hängigkeit hervorgebracht und innerhalb gesellschaftlicher Steuerungsprozesse eingesetzt werden (Asad, 2003; Casanova, 2011). Die aktuellen Debatten um Post-Säkularität, welche die Motive „klassischer“ Säkularisierungstheorien hinterfragen, greifen solche Perspektiven stellenweise auf (Dressler und Mandair, 2011). Während Habermas (2008) mit Post-Säkularität vorwiegend politische Übersetzungsmodi zwischen religiösen und nicht-religiösen Positionen diskutiert, fokussieren Baker und Beaumont (2011) aus geographischer Perspektive religiös-säkulare Kooperationsformen in gesellschaftspolitischen Handlungsfeldern. Demgegenüber erkunden Mandair und Dressler ,the various epistemological and political implications of the formation and codependency of secular and religious discourses" (2011:3). So verstanden kann das Post-Säkularitätskonzept für die vielgestaltigen Prozesse der Auslotung des Verhältnisses zwischen dem Säkularen, dem Politischen und dem Religiösen sensibilisieren und den Blick auf die Praktiken bestimmter „Grenzbereiche" richten. Wie wird bspw. der „Grenzbereich“ zwischen theologischen Angelegenheiten/Glaubensfragen und „säkularer" Kommunalpolitik praktiziert? Welche Praktiken können hierbei Grenzziehungen unterlaufen? Dabei erscheinen gewisse Formen jener „Grenzarbeit/-verschiebung“ als Elemente der Gouvernementalität des Dialogs - z.B. das „gleichzeitig-polyphone“ Sprechen als städtischer Repräsentant und „Katholik“, der von persönlichen Erfahrungen berichtet (vgl. Kapitel 5.4).

Nachfolgend wird nun versucht, governing through friendship konzeptionell weiter einzuordnen. An welche Technologien der Macht knüpft dieser Führungsmodus an? Welche Formen von Expertise und Wissen erlangen hierbei Wirksamkeit?

\subsection{Liberale Gouvernementalität}

„Fundamental in shaping the governmental rationalities of the West" (Rose, 1993:290), manifestieren sich liberale Regierungstechniken in der steten Frage nach einem ,Zuviel“ des Regierens (Foucault, 2005:181-182) und damit als selbst-befragende/-reflexive Formen politischer Einflussnahme (Rose, 1993:292, 1999; Rose und Miller, 1992). Letztere wird dadurch gleichzeitig beschränkt und neu re-installiert. Die liberale Mahnung, möglichst wenig zu beeinflussen, bezieht sich auf die Ökonomie genauso wie auf den Bereich der Familie, auf das Individuum - das durch seine Freiheit zu regieren ist (Rose, 2006:147) - als auch zunehmend auf die (re- ligiöse) Community/Gemeinschaft, deren Selbstregulierungskompetenzen anerkannt, allerdings durch Setzung von Anreizen in „gewünschte Richtungen“ (Rose, 1993:290, 1996) gelenkt werden sollen. Auch governing through friendship ist dem Kalkül einer Gouvernementalität unterstellt, innerhalb dessen Kräftefeld Führung keine Verordnung sein kann, sondern die Autonomie des „Geführten“ zu wahren sucht.

Die Dialog-Partizipanten konstituieren sich gegenseitig nicht nur als integrationspolitische Partner (Rodatz, 2012), sondern auch als Vertrauenspersonen. Im governing through friendship wird jene liberale Selbstreflexivität und Selbstbeschränkung - die Frage „Regieren wir euch zu viel?“” - zu einer moralischen Verpflichtung, und darüber zur emotionalisierten, bisweilen ritualisierten Praktik. Die „Führung unter Freunden“ erscheint dann auch nicht länger als Führung, insofern sich Führungsansprüche als wohlmeinende und fürsorgliche „Ratschläge“ übersetzen (vgl. Solberg, 2016). So wird der Dialog zudem de-politisiert, insofern sein Verlauf und seine Resultate sich als quasi-natürliche Prozesse eines hierarchiefreien „Miteinander-Sprechens“ präsentieren.

Ich deute governing through friendship als einen Aspekt/Modus liberaler Regierungskunst, der u.a. über die Frage zu erschließen ist, wie der liberale „Spagat“ einer Führung durch Involvierung/,Partnerwerdung“ konkret prozessiert wird. Governing through friendship befragt dabei - methodologisch gewendet - die liberalen ,formula of rule“ (Rose, 1993:283) nach deren Vermitteltheit in und durch Mikropraktiken und stellt die emotional-affektiven Aspekte jener gouvernementalen Grundsatzspagate heraus. Damit ließ sich darstellen, wie die den Dialog prägende und für liberale Regierung symptomatische Grundspannung einer Führung durch (angestrebte) Selbstführung in einem de-politisierten „Miteinander" persönlicher Bekanntschaften und über eine emotionalisierte Konstitution ,einander vertrauter“ Experten aufgehoben wird.

\section{3 Über-/lokale Koordinaten}

In Erlangen lassen sich durchaus besondere Bedingungen für die Entfaltung von governing through friendship vorfinden. So ist auf die lange Tradition eines dezidiert politisch forcierten/moderierten christlich-islamischen Dialogs zu verweisen. Zudem ist es der ,überschaubaren“ Größe Erlangens (108000 Einwohner) - in Kombination mit migrationsgeschichtlichen und stadtentwicklungsbezogenen Aspekten - zuzuschreiben, dass nur eine geringe Anzahl an für den Dialog relevanten Ansprechpartner/innen existiert (so gibt es lediglich zwei Moscheevereine!). Dies macht eine face-to-face-Politik des ,persönlichen Kennenlernens“ praktikabler. Nichtsdestotrotz könnten sich die beschriebenen Kommunikationsstrukturen (vergleichbar) auch in anderen Kontexten manifestieren, insofern auch andernorts ähnliche Deutungsmuster zirkulieren (das „Vertrauensmotiv“, die Emotionalität von Religion) und vergleichbare DialogTechnologien Anwendung finden (z.B. das integrationspoli- 
tische Aufgreifen „,christlich“-religiöser Expertise sowie interreligiöser Dialoge als „Begegnungsorte“ oder die Stärkung informeller (Bekanntschafts-) Netzwerke; vgl. Kap. 2-3). Politisch-geographische Ansätze stehen hier vor der Aufgabe, eine Sensibilität sowohl für „lokale Kartographien [gesellschaftlicher (Selbst-)Steuerungsprozesse]“ (Bröckling und Krasmann, 2010:40) als auch für deren Verschränkungen mit überlokalen Prozessen zu entwickeln (Rose et al., 2006).

Die Entfaltung von governing through friendship hängt teilweise mit dem Zugang auf „Islam“/Religion zusammen: Gerade das Prinzip eines ,freundschaftlich-vertrauten“ Verhältnisses, das in informell-bekanntschaftsbasierten (halbbis nicht-öffentlichen) Arbeitskreisen auszubilden sei, erscheint als kommunal-politische Antwort auf die pauschalisierenden, festgefahrenen und ,überhitzten“ „Islam“Debatten (Spielhaus, 2013) (und als Bearbeitungsmodus einer vermeintlichen Emotionalität von Religion). Gleichzeitig jedoch scheinen Motive wie „Begegnung“, „Respekt“ oder gar „Empathie“ auch allgemein in lokalen Integrationsund Vielfaltspolitiken programmatisch und zu Orientierungspunkten entsprechender Verwaltungspraktiken zu werden (Kiepenhauer-Drechsler, 2013; Fortier, 2007; De Wilde, 2015).

Die beschriebenen „Dialog“-Praktiken wie z.B. das Ratschläge-Geben (durch Experten/innen), das ,Sich-SelbstRelativieren" oder das moderierte gemeinsame Reflektieren persönlicher Erfahrungen sind grundsätzlich auch als allgemeine Elemente liberalen Regierens deutbar, die auch außerhalb ,islambezogener“ Dialogansätze relevant und z.B. mit Rationalitäten der Partizipation oder der Partnerschaft/Partnerwerdung verknüpft sein können (Solberg, 2016; Ott und Wrana, 2010).

Mit der Erlanger Fallstudie ließ sich aufzeigen, wie bestimmte „Theorien“, Verfahrensweisen und Formen von Expertise innerhalb einer Mikrophysik der Bekanntschaft und Empathie performativ werden. Die entsprechenden Machteffekte werden nun unter Zuhilfenahme des Experten-Motivs weiter diskutiert, wobei gerade die Praktiken des ,persönlichen Erfahrungsaustausches“" weitere Aufmerksamkeit erhalten sollen.

\section{4 „Territorien“ der Erfahrung: Die (post-säkularen) Experten/innen des governing through friendship}

Angelehnt an Rose und Miller (1992:175) ist der Experte jemand, der zwischen Regierungszielen (oder: gesellschaftlichen Ordnungsvorstellungen) und den Alltagswelten der Subjekte vermittelt und dafür lokale Taktiken der Überzeugung, des Anreizes, der Bildung und Ermächtigung entwickelt. Das Motiv des Experten empfinde ich als für meine Analyse passend, da es sich um eine Personifikation handelt, welche Expertentum ,at work“ in dessen körperlichen, emotional-affektiven Ausprägungen fasst und als etwas repräsentiert, dass auch über persönliche Interaktionen in lokalen Kontexten abläuft. Governing through friendship er- scheint mir hierbei als eine politische Einflussnahme durch einen bestimmten Typus des Experten: Nicht nur neutraler Technokrat - wie tendenziell bei Rose (1993; Rose und Miller, 1992) -, sondern gleichzeitig auch eine über die Jahre konstante Vertrauensperson. Diese Experten/innen schöpfen Autorität nicht nur aus zertifiziertem, sondern auch aus informellem, lokal-spezifischem Wissen, zudem aus persönlicher Erfahrung, Einfühlungsvermögen und aus der Selbstkritik. Ihre Bedeutung erwächst aus der Fähigkeit, vor Ort einen persönlichen Zugang zu einer religiösen Gruppe aufrechtzuerhalten - um dann innerhalb dieses Verhältnisses qua Wissen gestaltend einzugreifen.

So schreiben Rose und Miller: „Experts hold out the hope that problems of regulation can remove themselves from the disputed terrain of politics and relocate onto the tranquil yet seductive territory of truth“ (1992:188). Diese „ExpertenWahrheit" ist für liberales Regieren (ko-)konstitutiv. Sie entspricht im Fallbeispiel zur Erwachsenenbildung der dort angewandten „Theorie“, dass das Hinterfragen eigener Glaubenssätze den Gläubigen in seiner Persönlichkeitsbildung weiterbringt, kompetenter und selbstbewusster macht. Diese entwicklungstheoretisch und religionspädagogisch eingefärbten Theorien finden in Erlangen einen lokalen intellektuellen Nährboden in den christlich-theologischen Fachbereichen, im neuen Islamisch-theologischen Institut sowie im seit 2002 bestehenden Interdisziplinären Zentrum für Islamischen Religionsunterricht. Gerade mit diesen Institutionen stehen die Dialoginitiativen CIAG und FMGE im Austausch, was die Spezifik lokaler Topologien von Macht/WissenVerschränkungen aufscheinen lässt (Dornhof, 2012). ${ }^{7}$ Somit wird die „Theorie/Wahrheit“, Religionen würden sich durch kritische Selbstreflexion gesellschaftlich integrieren, wissenschaftlich legitimiert.

Zusätzlich gestützt werden diese „Theorien“ im obigen Beispiel kraft des Verweises auf eigene Erfahrungen des städtischen Vertreters als religiös bewegter Mensch/Katholik. Dieser zeigt sich als kompetent in religiöser Selbstführung - und wo sich Erfahrung mit wissenschaftlicher Theorie deckt, wird das Argument umso machtvoller. Im „Dialog-der-Vertrauten“ werden Konflikte also nicht nur auf das Territorium der (technischen) Wahrheit, sondern immer auch auf das Territorium der (persönlichen) Erfahrung überführt, auf welchem eine eigene Mikrophysik der Macht wirkt (Foucault, 2005). Governing through friendship zeigt sich durch diesen Austausch persönlicher Erfahrungen geprägt, wobei dieser Austausch zu einem Vehikel der Rationalisierung religiöser Identität(en) wird. Das Sprechen über eigene Erfahrungen und Empfindungen - vom steten Nachfragen flankiert, ob ,sich alle noch damit identifizieren, was diskutiert wird“" (Zitat, FMGE, 2016) - ist im Dia-

\footnotetext{
${ }^{7}$ Auch prägen die lokalen Erfahrungen mit der Etablierung ,islamischen“ Religionsunterrichts (Kerler, 2008) den Erlanger Dialog mit.
} 
logprojekt explizit erwünscht und Ausdruck des angestrebten Vertrauensverhältnisses.

Die „städtischen“ Vertreter/innen, die in/durch Allianzen mit „,christlichen“ und „muslimischen“ Vertretern/innen zu Experten/innen der Dialogführung werden, müssen sich im Kontext der auf Vertrauensbildung zielenden Vergemeinschaftungsmaßnahmen als religionssensibel bzw. „religiös musikalisch“ (Henkel, 2011) zeigen (d.h. als gläubig und/oder für religiös-spirituelle Erfahrungen offen). Sodann wird Ihnen zugesprochen, die angenommene Emotionalität und alltagsbestimmende Kraft von Glauben nachvollziehen und moderieren zu können. Der Moderator des Freundeskreises sprach zu den „muslimischen“ Gemeindevertretern/innen gleichzeitig als Repräsentant lokaler Politik und Verwaltung und als „Christ/Katholik“. Die Praxis des Dialogs produziert genau diese Überlagerungen. Seinen Appell an kritische Selbstreflexion eigener Glaubensansichten legitimierte er entsprechend zweigleisig: Letztere würde einen selbst als gläubigen Menschen (,erfrischend“) selbstsicherer/-bewusster machen (religiöses Argument) sowie gleichzeitig eine Grundlage für das Zusammenleben in pluralen Stadtgesellschaften sein (politisches Argument). Ich bezeichne diese Dialog-Experten/innen insofern als postsäkular, als deren Praxis in Prozesse der Re-Artikulation des Verhältnisses zwischen dem Religiösen und dem Säkularen eingebunden ist und hierbei Konturen, Geltungsbereiche und Wechselbeziehungen säkularer und religiöser Politiken und Praktiken re-konfiguriert (z.B. den ,passenden“ Ort und die gesellschaftspolitische Relevanz/Funktion theologischexegetischer Debatten im Kontext säkular-staatlicher Integrationspolitik neu auslotet).

Der Dialog zielt grundsätzlich auf die Selbstführung des religiösen Subjekts. Die „Anreizung“ von Selbstführung spiegelt sich bspw. in spezifischen Frageritualen wieder: „Was erwartet ihr („Muslime“) vom Projekt, von der Stadt, vom Dialog?“" (aggregierte Zitate städtischer Vertreter/innen, FMGE 2015-16). Diese Fragen eröffnen Möglichkeiten für Mitsprache und Gegenstimmen. Sie können gleichzeitig als Formung des selbstreflexiven Subjekts gedeutet werden (Tezcan, 2007:68; Schiffauer, 2008). Man möchte, dass die „muslimischen“ Gemeinden die an sie herangetragen Vorschläge selber wollen. Dafür ist man bereit, mit einem Projekt zu warten, bis die Bereitschaft dazu sich erst entwickelt (worauf man hofft). „Städtische“, „, christliche“ und „,muslimische" Vertreter/innen debattieren über Entwicklungspfade für den lokalen „Islam“, die - so die Logik - nicht verordnet werden können, Zeit brauchen. Der Dialog verschreibt sich - als „gute Lokalpolitik“ - dem Motiv des Geduldigen.

\section{5 Öffnung}

Und mit Geduld geht es in die Öffnung des Innenlebens. Die evangelische Erwachsenenbildung organisierte 2016 zusammen mit der städtischen Kulturinitiative „Comic-SalonErlangen“ eine Ausstellung zu religiösen Karikaturen. Ein
Ziel hierbei, so der Initiator, sei es, „muslimische“ Empfindungswelten besser zu verstehen: „Wir wissen da ja nichts“ (Interview 2015). Dazu müssten sich „Muslime“ und die Mehrheitsgesellschaft öffnen und über ihre Empfindungen sprechen. Man wolle bspw. zusammen mit den „muslimischen" Gemeinden ergründen, welche (religiös, sozial, politisch aufgeladenen) Gefühle es sind, die bei der Betrachtung religiöser Karikaturen aufkommen. Hierbei würden sich viele Personen über sich selbst im Unklaren sein. Auf der Vernissage wurde argumentiert, dass religiöse Emotionen zukünftig an Relevanz gewinnen würden und deshalb frühzeitig gemeinsam behandelt werden sollten (Interview 2015; Beobachtungen 2016).

Das religiöse Subjekt wird hier auf ein Übungsfeld geführt, um die Offenlegung der eigenen Gefühlswelten zu trainieren - für die ,innere Sicherheit“ des Einzelnen und der Gemeinschaft. Die Experten/innen des Dialogs erhalten die Aufgabe, über ihre eigenen Erfahrungen zu reden, damit die Öffnung des „Gegenübers“ zu plausibilisieren und Raum für die Behandlung sensibler Themen zu schaffen. Der Fokus auf das Innenleben des Subjekts und somit die Intimisierung des Dialogs werden dabei mit den Motiven von Freundschaft und Vertrauen begründbar. Hajatpour, Lehrstuhlinhaber am 2012 etablierten Islamisch-theologischen Institut und intellektueller Mitstreiter des Projekts, ,islamische“ Subjektivitäten entlang der Rationalität des (interreligiösen) Dialogs auszurichten, schreibt:

„Mit dem Dialog erreichen wir das Innere des Mitmenschen, die andere Erfahrung, darüber hinaus verleiht er der eigenen Erfahrung eine kritische Selbstreflexion. Dialog ist daher die Fähigkeit, sich zu öffnen und seine Selbstverständlichkeiten in Frage zu stellen [... ]“ (2005:21)

Subjekte sind aufgerufen, den „Anderen“ als jemanden zu schätzen, der ihnen neue Perspektiven auf sich selbst ermöglicht sowie bestimmte Emotionen gegenüber sich selbst und dem „Anderen“ zu entwickeln (De Wilde, 2015). Im Dialog moderiert die religionssensible Stadt zusammen mit Religions-Experten/innen derartige (Selbst-)Öffnungsprozesse. Der Dialog erhält hierbei Impulse aus dem Wissensfeld der Religionspädagogik. Diese, so Harry Behr (langjährig am Erlanger Zentrum für Islamischen Religionsunterricht und teils im interreligiösen Dialog aktiv, vielen Dialogaktiven bekannt), müsse dem Subjekt helfen, „sich selbst seiner eigenen Absichten bewusster zu werden“ (2011:17). Dies ermögliche, „Kontrolle über das eigene Verhalten zu erlangen und zu lernen, sich mit seinen Mitmenschen [...] auszutauschen. Jede Unaufrichtigkeit und Unklarheit in dieser Verständigung führt zu [... Konflikten“" (ebd.: 17). Diese Sätze erscheinen als Programmatik eines besonderen Aspekts dessen, was ich Gouvernementalität der Freundschaft nenne. 


\section{Resümee}

Im vorliegenden Beitrag rekonstruierte ich anhand empirischer Studien zum Umgang mit „Islam“ in der Stadt Erlangen die Operationsweisen einer spezifischen Gouvernementalität. Ich rekonstruierte governing through friendship als ein Regieren durch eine emotional aufgeladene Vergemeinschaftungspraxis. Die zugrundeliegende politische Technologie des Dialogs wird gegenwärtig in vielen Städten in Deutschland als Politik der „Augenhöhe“ programmatisch. Ich zeigte, wie der Dialog sowohl an überlokale Problematisierungen als auch an lokalspezifische Themenfelder, Wissensquellen und Orte geknüpft ist. Die DialogTechnologie zielt auf die Herstellung von Nähe und Vertrauen und setzt auf konstante Kontaktpartner, „face-to-face“Beziehungen, kurze Kommunikationswege und persönlichinformelle Netzwerke. Der Ort dieser Praktiken ist in Erlangen - wie in anderen deutschen Großstädten - ein integrationspolitisch modellierter interreligiöser Dialog. In dessen Feld konstituiert sich die Subjektposition des „Vertrauten“, die mit spezifischen Machteffekten einhergeht. Die Technologien des Dialogs rekurrieren dabei vielfach auf lokale Identität(en) und fokussieren auf lokale Vergemeinschaftung ,,vor Ort“.

Damit verbunden zeigte sich der Dialog mit „Muslimen“ als eine religionssensible Technologie, in welcher (a) Wissen über Religionen sowie (b) eigene Religiosität und religiöse Sensibilität an Wert gewinnen. Die sich auf diesen Wissensformen begründenden Gespräche über Religion und Glaubenspraxis werden zu Elementen des angestrebten verständnisorientierten Zugangs auf die „muslimische“ Bevölkerung, der mit besonderen Techniken der Neu-Ausrichtung ,muslimischer" Identitäten einhergeht. Stadtpolitiken setzen auf einen Dialog, in welchem spezifisch sensibilisierte und interessierte postsäkulare Experten/innen vermittels religiöser Musikalität Vertrauensarbeit zwischen Stadt(-gesellschaft), Kirchen und „muslimischen“ Gemeinden dirigieren und unter Bezugnahme auf persönliche Erfahrungen ein politischpädagogisches Projekt der Involvierung von „Muslimen“ in die zivilgesellschaftliche Ordnung begründen.

Mit der Figur des post-säkularen Experten lenkte die Studie die Aufmerksamkeit auf den Bedeutungsgewinn und den Einsatz religiöser Kompetenzen und Sensibilitäten in integrationspolitischen Kontexten. Die Post-SäkularitätsPerspektive hob die Durchlässigkeit der Grenzen zwischen säkular-politischen und religiösen Aushandlungsbereichen hervor (Cloke und Beaumont, 2012) und ließ andenken, wie diese Grenzüberschreitungen zu Bedingungen von Steuerungsprozessen werden. Dabei vermochte es ein auf Foucaults Machtanalytik rekurrierender, politisch-geographischer Blick auf ,lokale Rationalitätsmuster und Praktiken des Regierens" (Bröckling und Krasmann, 2010:26) aufzuzeigen, wie die Re-Konfigurierung ,muslimischer" Identität(en), die emotionalisierte Praxis lokaler Vergemeinschaftung, die Bedeutung religionsbezogener Exper- tise und jene post-säkularen Grenzverschiebungen allesamt zusammenhängen. Diese Analyse politischer Geographien des interreligiösen/interkulturellen Dialogs lieferte mehrere Diskussionsimpulse. Die Illustration der besonderen Bedeutung des Städtisch-Lokalen für den Dialog als auch allgemein die Rekonstruktion lokaler Spezifika von Regierungsprozessen erscheinen für politisch-geographische Ansätze als auch für die Gouvernementalitätsforschung von Interesse. Beide erhalten zudem Anregungen zur Diskussion der Bedeutung von Emotionalität im Kontext politischer Steuerung. Ferner brachte die Analyse von Regierungspraktiken im Grenzraum zwischen säkular-staatlichen (Integration)Politiken und religiösen Deutungsbereichen die Debatte um Post-Säkularität mit politisch-geographischen Fragen gesellschaftlicher Steuerungsprozesse zusammen.

Datenverfügbarkeit. Im Sinne des Schutzes vertraulicher und persönlicher Informationen und des Schutzes von Persönlichkeitsrechten liegen die durch qualitative Methoden erhobenen Daten nicht öffentlich zugänglich vor. Sie sind jedoch am Institut für Geographie der Friedrich-Alexander-Universität Erlangen-Nürnberg archiviert und können auf Nachfrage an den Autor in einem dem Nachfrageinteresse angemessenen Umfang (partiell oder vollständig) eingesehen werden.

Interessenkonflikt. Der Autor erklärt, dass kein Interessenkonflikt besteht.

Danksagung. Ich möchte mich bei den zwei anonymen Gutachter/innen, den Special-Issue-Editoren Georg Glasze und Thomas Schmitt sowie bei Benedikt Korf für zahlreiche hilfreiche Kommentare bedanken. Der vorliegende Beitrag basiert auf Forschungsarbeiten im Kontext eines durch die Deutsche Forschungsgemeinschaft (DFG) geförderten Projekts zu „Konfigurierungen von Islam und Muslimen auf lokaler Ebene in Deutschland“.

Edited by: Benedikt Korf

Reviewed by: two anonymous referees

\section{Literatur}

Amir-Moazami, S.: Pitfalls of consensus-orientated dialogue: the German Islam Conference (Deutsche Islam-Konferenz), Approaching Religion, 1, 2-15, 2011a.

Amir-Moazami, S.: Dialogue as a governmental technique: managing gendered Islam in Germany, Feminist Rev., 98, 9-27, $2011 b$.

Asad, T.: Formations of the Secular. Christianity, Islam, Modernity, Stanford-University-Press, Stanford, 2003.

Baker, C. R. und Beaumont, J. (Hg.): Postsecular cities, Religious space, theory and practice, Continuum, London, 2011.

Bargetz, B. und Sauer, B.: Politik, Emotionen und die Transformation des Politischen. Eine feministisch-machtkritische Perspekti- 
ve, Österreichische Zeitschrift für Politikwissenschaft (ÖZP), 39, 141-155, 2010.

Behr, H. H.: Integration, Islamunterricht und Koran, Zeitschrift für die Religionslehre des Islam, 5, 7-19, 2011.

Bröckling, U. und Krasmann, S.: Ni methode, ni approche, in: Diskursanalyse meets Gouvernementalitätsforschung - Perspektiven auf das Verhältnis von Subjekt, Sprache, Macht und Wissen, Herausgeber: Angermüller, J. und van Dyk, S., Campus, Frankfurt/New York, 23-43, 2010.

Brökling, U., Krasmann, S. und Lemke, T. (Hg.): Governmentality: Current issues and future challenges, Routledge, London, 2011.

Burchell, G.: Liberal government and techniques of the self, Econ. Soc., 22, 267-282, 1993.

Campbell, E.: The emotional life of governmental power, Foucault Studies, 9, 35-53, 2010.

Casanova, J.: The Secular, Secularizations, Secularisms, in: Rethinking Secularism, Herausgeber: Calhoun C. J., Juergensmeyer, M. und Van Antwerpen, J., Oxford University Press, Oxford/New York, 54-75, 2011.

Cloke, P. und Beaumont, J.: Geographies of postsecular rapprochement in the city, Prog. Hum. Geog., 37, 27-51, 2012.

Crouch, C.: Post-Democracy, John Wiley \& Sons, Cambridge, 2004.

D'Aoust, A. M.: Ties that Bind? Engaging Emotions, Governmentality and Neoliberalism: Introduction to the Special Issue, Global Society, 28, 267-276, 2014.

De Wilde, M.: Profound Coziness: Affective Citizenship and the Failure to Enact Community in a Dutch Urban Neighborhood, in: Die Ambivalenz der Gefühle. Über die verbindende und widersprüchliche Sozialität von Emotionen, Herausgeber: Kleres, J. und Albrecht, Y., Springer, Wiesbaden, 125-143, 2015.

DIK (Deutsche Islamkonferenz): Drei Jahre Deutsche Islam Konferenz (DIK) 2006-2009: Muslime in Deutschland - deutsche Muslime, Online unter: http://www.deutsche-islam-konferenz. de/SharedDocs/Anlagen/DIK/DE/Downloads/

LenkungsausschussPlenum/dik-broschuere-download.pdf; jsessionid=81199E7BFA6B44CDB1F2F58246D13A37.1_ cid368?_blob=publicationFile (letzter Aufruf: 11. Juli 2016), 2009.

Dornhof, S.: Rationalities of dialogue, Curr. Sociol., 60, 382-398, 2012.

Dressler, M. und Mandair, A.-P. S. (Hg.): Secularism and ReligionMaking, Oxford University Press, Oxford/New York, 2011.

Fortier, A. M.: Too close for comfort: loving thy neighbour and the management of multicultural intimacies, Environ. Plann. D, 25, 104-119, 2007.

Foucault, M.: Überwachen und Strafen, Die Geburt des Gefängnisses, Suhrkamp, Frankfurt/Main, 1977.

Foucault, M.: Sicherheit, Territorium, Bevölkerung: Geschichte der Gouvernementalität I - Vorlesungen am College de France I977I978, Suhrkamp, Frankfurt/Main, 2004 [1977-78].

Foucault, M.: Die Geburt der Biopolitik: Geschichte der Gouvernementalität II - Vorlesung am College de France I978-I979, Suhrkamp, Frankfurt/Main, 2004 [1978-79].

Foucault, M.: Analytik der Macht, Suhrkamp, Frankfurt/Main, 2005.

Füller, H. und Marquardt, N.: Gouvernementalität in der humangeographischen Diskursforschung, in: Handbuch Diskurs und Raum,
Herausgeber: Glasze, G. und Mattissek, A., Transcript, Bielefeld, 83-106, 2009.

Füller, H. und Marquardt, N.: Die Sicherstellung von Urbanität. Innerstädtische Re strukturierung und soziale Kontrolle in Downtown Los Angeles, Dampfboot Verlag, Münster, 2010.

Ghatak, S. und Abel, A. S.: Power/Faith: Governmentality, Religion, and Post-Secular Societies, International Journal of Politics, Culture, and Society, 26, 217-235, 2013.

Habermas, J.: Die Dialektik der Säkularisierung, Blätter für deutsche und internationale Politik, 4/2008, Online unter: http://www.eurozine.com/articles/2008-04-15-habermas-de. html (letzter Aufruf: 14. November 2016), 2008.

Hajatpour, R.: Identität und Anerkennung: Religiöse Erfahrung ein Prozess der Selbstreflexion, UNI.VERS - Das Magazin der Otto-Friedrich-Universität Bamberg, 2005, 19-21, 2005.

Henkel, R.: Are Geographers religiously unmusical? Positionalities in geographical research on religion, Erdkunde, 65, 389-399, 2011.

Hutta, J. S.: The affective life of semiotics, Geogr. Helv., 70, 295 309, https://doi.org/10.5194/gh-70-295-2015, 2015.

Isik, T. und Schmitz, S. (Hg.): Muslimische Identitäten in Europa. Dispositive im gesellschaftlichen Wandel, Transcript, Bielefeld, 2015.

Jessop, B.: From micro-powers to governmentality: Foucault's work on statehood, state formation, statecraft and state power, Polit Geogr., 26, 34-40, 2007.

Kerler, W.: Zwei Seiten einer Medaille. Der Islamunterricht in Berlin und Erlangen im Vergleich, Herausgegeben vom Zentralinstitut für Regionalforschung Erlangen, Erlangen, 2008

Kiepenhauer-Drechsler, B.: Vielfalt plus Zusammenhalt. Eine ethnographische Perspektive auf die Praxis Berliner Integrationspolitik, Bielefeld, 2013.

Klinkhammer, G., Frese, H. L., Satilmis, A. und Seibert, T. (Hg.): Interreligiöse und interkulturelle Dialoge mit MuslimInnen in Deutschland. Eine quantitative und qualitative Studie, Universität Bremen, Bremen, 2011.

Koenig, M.: Staatsbürgerschaft und religiöse Pluralität in postnationalen Konstellationen: Zum institutionellen Wandel europäischer Religionspolitik am Beispiel der Inkorporation muslimischer Immigranten in Großbritannien, Frankreich und Deutschland, Universität Marburg, Marburg, 2003.

KQI (Kommunaler Qualitätszirkel zur Integrationspolitik): Empfehlungen des kommunalen Qualitätszirkels zur Integrationspolitik ,Umgang mit religiöser Vielfalt - Handreichung für die kommunale Praxis“, Online unter: http://www.stuttgart.de/img/mdb/ item/385012/82577.pdf (letzter Aufruf: 11. November 2016), 2012.

Kuhla, K. und Szukitsch, Y. (Netzwerk-Integration-Bayern): Religion in der kommunalen Integrationsarbeit, Herausgegeben von „VIA Bayern e.V. - Verband für interkulturelle Arbeit“", Online unter: http://www.via-bayern.de/NIB/bilder/nib_ heft_religion.pdf (letzter Aufruf: 11. November 2016), 2011.

Mahmoud, S.: Secularism, Hermeneutics, and Empire: The Politics of Islamic Reformation, Public Culture, 18, 323-347, 2006.

Mandair, A.-P. S. und Dressler, M.: Introduction: Modernity, Religion-Making, and the Postsecular, in: Secularism and Religion-Making, Herausgeber: Dressler, M. und Mandair, A.-P. S., Oxford University Press, Oxford/New York, 3-37, 2011. 
Marquardt, N.: Michel Foucault - Gouvernementalität und Stadt, in: Handbuch Kritische Stadtgeographie, Herausgeber: Belina, B., Naumann, M. und Strüver, A., Westfälisches Dampfboot, Münster, 20-25, 2014.

Marquardt, N.: Das Regieren von Emotionen in Räumen des betreuten Wohnens, Geogr. Helv., 70, 175-184, https://doi.org/10.5194/gh-70-175-2015, 2015.

Mavelli, L.: Between Normalisation and Exception: The Securitisation of Islam and the Construction of the Secular Subject, Millennium: Journal of International Studies, 41, 159-181, 2013.

Michels, C.: Researching affective atmospheres, Geogr. Helv., 70, 255-263, https://doi.org/10.5194/gh-70-255-2015, 2015.

Modood, T., Triandafyllidou, A., und Zapata-Barrero, R. (Hg.): Multiculturalism, Muslims and Citizenship. A European approach, Routledge, London/New York, 2006.

Mouffe, C.: Über das Politische. Wider die kosmopolitische Illusion, Suhrkamp, Frankfurt/Main, 2007.

Müller, M.: Mittendrin statt nur dabei: Ethnographie als Methodologie in der Humangeographie, Geogr. Helv., 67, 179-184, https://doi.org/10.5194/gh-67-179-2012, 2012.

Müller, M.: More-than-representational political geographies, in: The Wiley-Blackwell Companion to Polit. Geogr., Herausgeber: Agnew, J., Mamadouh, V., Secor, A., und Sharp, J., WileyBlackwell, Oxford, 409-424, 2015.

Peter, F.: Political Rationalities, Counter-terrorism and Policies on Islam in the United Kingdom and France, in: The Social Life of Anti-Terrorism Laws, Herausgeber: Eckerts, J. M., Transcript, Bielefeld, 79-108, 2008.

Peter, F.: Welcoming Muslims into the nation: Tolerance, politics and integration in Germany, in: Muslims in the West after 9/11: Religion, Politics and Law, Herausgeber: Cesari, J., Routledge, London und New York, 119-144, 2010.

Rancière, J.: On the Shores of Politics, Verso, London/New York, 1995.

Rochdi, A.: Islamischer Religionsunterricht als Motor für die Entwicklung islamischer Theologie in Deutschland, Zeitschrift für die Religionslehre des Islam, 2, 21-26, 2008.

Rodatz, M.: Produktive „Parallelgesellschaften“. Migration und Ordnung in der (neoliberalen) „Stadt der Vielfalt“, Behemoth. A Journal on Civilisation, 5, 70-103, 2012.

Rommelspacher, B.: Identitätspolitik in Deutschland zwischen Islamisierung und (Re-) Christianisierung, in: Islam und Diaspora. Analysen zum muslimischen Leben in Deutschland aus historischer, rechtlicher sowie migrations- und religionssoziologischer Perspektive, Herausgeber: Ceylan, R., Lang, Frankfurt/Main, 205-220, 2012.

Rose, N.: Government, authority and expertise in advanced liberalism, Econ. Soc., 22, 283-299, 1993.

Rose, N.: The Death of the social? Re-figuring the territory of government, Econ. Soc., 25, 327-356, 1996.

Rose, N.: Powers of Freedom. Reframing political thought, Cambridge Univesity Press, Cambridge, 1999.

Rose, N.: Governing 'advanced' liberal democracies, in: The Anthopology of the state. A reader, Herausgeber: Sharma, A. und Gupta, A., Blackwell Publishing, Malden/Oxford/Victoria, 144163, 2006.

Rose, N. und Miller, P.: Political Power beyond the State: Problematics of Government, The British Journal of Sociology, 43, 173205, 1992.
Rose, N., O’Malley, P. und Valverde, M.: Gouvernementality, Annual Review of Law and Social Science, 2, 83-104, 2006.

Schatzki, T. R.: The site of the social: A philosophical account of the constitution of social life and change, Pennsylvania State University Press, University Park, 2002.

Schiffauer, W.: Der Islam als Civil Religion. Eine deutsche Geschichte, in: Fremde in der Stadt, Herausgeber: Schiffauer, W. Suhrkamp, Frankfurt/Main, 50-70, 1997.

Schiffauer, W.: Verwaltete Sicherheit - Präventionspolitik und Migration, in: Migrationsreport 2006: Fakten - Analysen - Perspektiven, Herausgeber: Bommes, M. und Schiffauer, W., Campus Verlag, Frankfurt/Main, 113-164, 2006.

Schiffauer, W.: Zur Konstruktion von Sicherheitspartnerschaften, in: Migrationsreport 2008. Fakten - Analysen - Perspektiven, Herausgeber: Bommes, N. und Krüger-Potratz, M., Campus Verlag, Frankfurt/New York, 205-237, 2008.

Schreiber, V.: Fraktale Sicherheiten. Eine Kritik der kommunalen Kriminalprävention, Transcript, Bielefeld, 2011.

Schubert, K. und Meyer, H. (Hg.): Politik und Islam, SpringerVerlag, Wiesbaden, 2011.

Schurr, C.: Towards an Emotional Electoral Geography: The Performativity of Emotions in Electoral Campaigning in Ecuador, Geoforum, 49, 114-126, 2013.

Silvestri, S.: Public policies towards Muslims and the institutionalization of „Moderate Islam“ in Europe: some critical reflections, in: Muslims in 21st century Europe: Structural and cultural perspectives, Herausgeber: Triandafyllidou, A., Routledge, London/New York, 45-59, 2010.

Solberg, J.: The art of not governing too much in vocational rehabilitation encounters, in: Studies of Discourse and Governmentality: New perspectives and methods, Herausgeber: McIlvenny, P., Zhukova Klausen, J. und Band Lindegaard, L., John Benjamins publishing company, Amsterdam/Philadelphia, 119-149, 2016.

Spielhaus, R.: Vom Migranten zum Muslim und wieder zurück Die Vermengung von Integrations- und Islamthemen in Medien, Politik und Forschung, in: Islam und die deutsche Gesellschaft, Herausgeber: Meyer, H. und Halm, D., Springer, Wiesbaden, 169-194, 2013.

Stadt Erlangen: Mitteilung zur Kenntnis. Bericht über mosaik - Interkulturelle Anlaufstelle nicht nur für muslimische Familien, Jugendhilfeausschuss der Stadt Erlangen, 29. Juli 2009, Online unter: http://ratsinfo.erlangen.de/to0040.php? ksinr $=1778092 \&$ toselect $=2003150$ (letzter Aufruf: 11. Juli 2016), 2009.

Strüver, A.: „Ich war lange illegal hier, aber jetzt hat mich die Grenze übertreten“ - Subjektivierungsprozesse transnational mobiler Haushaltshilfen, Geogr. Helv., 68, 191-200, https://doi.org/10.5194/gh-68-191-2013, 2013.

Tezcan, L.: Interreligiöser Dialog und politische Religionen, Bundeszentrale für politische Bildung, Online unter: http://www.bpb.de/apuz/29643/ interreligioeser-dialog-und-politische-religionen? $\mathrm{p}=$ all (letzter Aufruf: 9 Mai 2016), 2006.

Tezcan, L.: Kultur, Gouvernementalität der Religion und der Integrationsdiskurs, in: Konfliktfeld Islam in Europa, Herausgeber: Wohlrab-Sahr, M. und Tezcan, L., Nomos Verlag, Baden-Baden, 51-74, 2007.

Tezcan, L.: Operative Kultur und die Subjektivierungsstrategien in der Integrationspolitik, in: Wider den Kulturenzwang: Migration, 
Kulturalisierung und Weltliteratur, Herausgeber: Ezli, Ö., Kimmich, D. und Werberger, A., Transcript, Bielefeld, 47-80, 2009.

Tezcan, L.: Der säkulare Muslim. Zur Generierung einer Kategorie im Kontext der Deutschen Islam Konferenz, in: Migrationsreport 2010. Fakten - Analysen - Perspektiven, Herausgeber: KrügerPotratz, M. und Schiffauer, W., Campus Verlag, Frankfurt /New York, 83-108, 2011a.

Tezcan, L.: Spielarten der Kulturalisierung, Zeitschrift für Kulturphilosophie, 2, 357-376, $2011 \mathrm{~b}$.

Tezcan, L.: Das muslimische Subjekt. Verfangen im Dialog der Deutschen Islamkonferenz, Konstanz University Press, Konstanz, 2012.
Tuori, S.: The politics of multicultural encounters - Feminist postcolonial perspectives, Åbo Akademi University Press, Åbo, 2009.

Wrana, D. und Ott, M.: Gouvernementalität diskursiver Praktiken. Zur Methodologie der Analyse von Machtverhältnissen am Beispiel einer Massnahme zur Aktivierung von Erwerbslosen, in: Diskursanalyse meets Gouvernementalitätsforschung. Perspektiven auf das Verhältnis von Subjekt, Sprache, Macht und Wissen, Herausgeber: Angermüller, J. und van Dyk, S., Campus Verlag, Frankfurt/Main, 155-182, 2010. 